\title{
A THEOREM OF COMPLETENESS FOR COMPLEX ANALYTIC FIBRE SPACES
}

\author{
BY \\ K. KODAIRA and D. C. SPENCER \\ The Institute for Advanced Study and Princeton University
}

\section{Introduction}

We begin by recalling several definitions, introduced in the authors' paper [3], concerning complex analytic families of complex manifolds.

By a complex analytic fibre space we mean a triple $(\boldsymbol{\vartheta}, \boldsymbol{\sigma}, \boldsymbol{M})$ of connected complex manifolds $\mathfrak{V}, M$ and a holomorphic map $\varpi$ of $\mathfrak{V}$ onto $M$. A fibre $\varpi^{-1}(t)$, $t \in M$, of the fibre space is singular if there exists a point $p \in \sigma^{-1}(t)$ such that the rank of the jacobian matrix of the map $\varpi$ at $p$ is less than the dimension of $M$.

DEFINITION 1 . We say that $\vartheta \stackrel{\pi}{\rightarrow} M$ is a complex analytic family of compact, complex manifolds if $(\vartheta, \varpi, M)$ is a complex analytic fibre space without singular fibres whose fibres are connected, compact manifolds and whose base space $M$ is connected.

With reference to a complex manifold $V_{0}=\varpi^{-1}(0), 0 \in M$, we call any $V_{t}=\varpi^{-1}(t)$, $t \in M$, a deformation of $V_{0}$ and we call $\mathfrak{v} \stackrel{\varpi}{\rightarrow} M$ a complex analytic family of deformations of $V_{0}$.

DEFINITION 2. A complex analytic family $\vartheta^{\rightarrow} M$ of compact, complex manifolds is (complex analytically) complete at the point $t \in M$ if, for any complex analytic family $\mathcal{U} \underset{\boldsymbol{\pi}}{\rightarrow} N$ such that $\pi^{-1}(0)=\varpi^{-1}(t)$ for a point $0 \in N$, there exist a holomorphic map $s \rightarrow t(s), t(0)=t$, of a neighborhood $U$ of 0 on $N$ and a holomorphic map $g$ of $\pi^{-1}(U)$ into $\vartheta$ which maps each fibre $\pi^{-1}(s), s \in U$ of $\mathcal{W}$ biregularly onto $\varpi^{-1}(t(s))$. The complex analytic family $\vartheta^{\star} \rightarrow M$ is called (complex analytically) complete if it is (complex analytically) complete at each point $t$ of $M$. 
Let $\vartheta \stackrel{\varpi}{\rightarrow} M$ be a complex analytic family of compact, complex manifolds and let $V_{t}=\varpi^{-1}(t)$ be the fibre of $\mathfrak{V}$ over $t \in M$. Denote by $\Theta_{t}$ the sheaf over $V_{t}$ of germs of holomorphic vector fields, and denote by $\left(T_{M}\right)_{t}$ the (complex) tangent space of $M$ at the point $t$. Of fundamental importance in the study of the deformation of complex structure is the complex linear map

$$
\varrho_{t}:\left(T_{M}\right)_{t} \rightarrow H^{1}\left(V_{t}, \Theta_{t}\right)
$$

which measures the magnitude of dependence of the complex structure of the fibre $V_{t}$ on the parameter $t$ (see [3], Sections 5 and 6). A definition of $\varrho_{t}$ will be given below (see formula (9)). For a tangent vector $v \in\left(T_{M}\right)_{t}$ the image $\varrho_{t}(v) \in H^{1}\left(V_{t}, \Theta_{t}\right)$ is called the infinitesimal deformation of $V_{t}$ along $v$.

Our purpose is to prove the following theorem:

THEOREM. Let $\mathfrak{v} \stackrel{\varpi}{\rightarrow} M$ be a complex analytic family of compact, complex manifolds and suppose that, for some point $t \in M$, the map $\varrho_{t}:\left(T_{M}\right)_{t} \rightarrow H^{1}\left(V_{t}, \Theta_{t}\right)$ is surjective. Then $\mathfrak{*} \rightarrow M$ is (complex analytically) complete at $t$.

The proof of this theorem is elementary, in particular it makes no use of the theory of harmonic differential forms.

We remark that the question remains open whether $\mathfrak{v} \stackrel{\varpi}{\rightarrow} M$ is differentiably complete at $t \in M$ (in the sense of [3], Definition 1.7) if the map $\varrho_{t}:\left(T_{M}\right)_{t} \rightarrow H^{1}\left(V_{t}, \Theta_{t}\right)$ is surjective; in particular, Problem 6, Section 22 of [3], remains unsolved. If we assume the additional condition that $H^{2}\left(V_{t}, \Theta_{t}\right)=0$ at this particular point $t$, then it can be proved, by the method of harmonic differential forms, that $\mathfrak{v} \stackrel{\mathbb{W}}{\rightarrow} M$ is differentiably complete at $t$ (see Kodaira [2]).

In [3] the authors constructed several simple examples of complex analytic families of compact, complex manifolds, namely:

(1) family of complex tori of arbitrary dimension $n$;

(2) family $\vartheta_{n, h}$ of all non-singular hypersurfaces of order $h$ on complex projective $n$-space $(n \geqslant 2, h \geqslant 2)$;

(3) family of non-singular hypersurfaces on abelian varieties of arbitrary dimen:siön $n \geqslant 2$;

(4) family of compact Hopf surfaces.

It was shown in Section 18 of [3], on the basis of special properties of the families, that the families (1) and (2) are complex analytically complete, except for the case $n=2, h=4$ of (2) in which the map $\varrho_{t}$ is not surjective. The (complex ana- 
lytic) completeness of all four families (except the case $n=2, h=4$ of (2) in which the family is not complete) now follows at once from the above theorem.

We remark that each of the above families (except $\mathfrak{V}_{2.4}$ ) is differentiably complete (see [3]).

\section{Complex analytic completeness (proof of the theorem)}

Let $\mathfrak{v} \stackrel{\varpi}{\rightarrow} M$ be a complex analytic family which satisfies the hypothesis of our theorem, namely that, for some point $0 \in M$, the map

$$
\varrho_{0}:\left(T_{M}\right)_{0} \rightarrow H^{1}\left(V_{0}, \Theta_{0}\right)
$$

is surjective, where $V_{0}=\varpi^{-1}(0)$ is the fibre over the point $0 \in M$. Given an arbitrary complex analytic family $\mathcal{W} \stackrel{\pi}{\rightarrow} N$ such that $\pi^{-1}(0)=V_{0}$ for a point $0 \in N$, we must show that there exist a holomorphic map $s \rightarrow t(s), t(0)=0$, of a neighborhood $U$ of 0 on $N$ into $M$ and a holomorphic map $g$ of $\mathcal{W} \mid U=\pi^{-1}(U)$ into $\vartheta$ which maps each fibre $\pi^{-1}(s), s \in U$, of $W$ biregularly onto $\varpi^{-1}(t(s))$.

First we fix our notations. We denote by $t$ a point $\left(t_{1}, t_{2}, \ldots, t_{m}\right)$ on the space $\mathbf{C}^{m}$ of $m$ complex variables and by $s$ a point $\left(s_{1}, s_{2}, \ldots, s_{l}\right)$ on $\mathbf{C}^{l}$. We define

$$
\begin{aligned}
& |t|=\max _{r}\left|t_{r}\right|, \\
& |s|=\max _{r}\left|s_{r}\right| .
\end{aligned}
$$

Similarly we denote by $z_{i}$ a point $\left(z_{i}^{1}, z_{i}^{2}, \ldots, z_{i}^{n}\right)$, by $\zeta_{i}$ a point $\left(\zeta_{i}^{1}, \ldots, \zeta_{i}^{n}\right)$, and let

$$
\begin{aligned}
& \left|z_{l}\right|=\max _{\alpha}\left|z_{i}^{\alpha}\right|, \\
& \left|\zeta_{i}\right|=\max _{\alpha}\left|\zeta_{i}^{\alpha}\right| .
\end{aligned}
$$

If

$$
f: s \rightarrow f(s)=\left(f^{1}(s), \ldots, f^{\alpha}(s), \ldots, f^{n}(s)\right)
$$

is a holomorphic map of a domain $\{s|| s \mid<\varepsilon\}$ into $\mathbf{C}^{n}$, we write the power series expansion of $f^{\alpha}(s)$ in the form

$$
f^{\alpha}(s)=f_{0}^{\alpha}+f_{1}^{\alpha}(s)+\cdots+f_{\mu}^{\alpha}(s)+\cdots,
$$

where $f_{\mu}^{\alpha}(s)$ is a homogeneous polynomial in $\left(s_{1}, s_{2}, \ldots, s_{l}\right)$ of degree $\mu$. Moreover, letting

we write

$$
f_{\mu}(s)=\left(f_{\mu}^{1}(s), \ldots, f_{\mu}^{\alpha}(s), \ldots, f_{\mu}^{n}(s)\right)
$$

$$
f(s)=f_{0}+f_{1}(s)+\cdots+f_{\mu}(s)+\cdots
$$


and call this the power series expansion of the vector-valued holomorphic function $f(s)$.

We may assume the following:

i) $M$ is a polycylinder: $M=\{t|| t \mid<1\}$ and $V_{0}=\varpi^{-1}(0)$.

ii) $\boldsymbol{v}$ is covered by a finite number of coordinate neighborhoods $\boldsymbol{u}_{i}$. Each $\boldsymbol{u}_{i}$ is covered by a system of holomorphic coordinates $\left(\zeta_{i}, t\right)$ such that $\varpi\left(\zeta_{i}, t\right)=t$ and

$$
u_{i}=\left\{\left(\zeta_{i}, t\right)|| \zeta_{i}|<1,| t \mid<1\right\}
$$

(We indicate by $\left(\zeta_{i}, t\right)$ a set of $n+m$ complex numbers $\zeta_{i}^{1}, \ldots, \zeta_{i}^{n}, t_{1}, \ldots, t_{m}$ and the point on $u_{i}$ with the coordinates $\left(\zeta_{i}^{1}, \ldots, \zeta_{i}^{n}, t_{1}, \ldots, t_{m}\right)$.)

iii) $\left(\zeta_{t}, t\right)$ coincides with $\left(\zeta_{k}, t\right)$ if and only if

$$
\zeta_{i}=g_{i k}\left(\zeta_{k}, t\right)
$$

where $g_{i k}\left(\zeta_{k}, t\right)$ is a vector-valued holomorphic function of $\left(\zeta_{k}, t\right)$ defined on $u_{k} \cap u_{i}$.

iv) $N$ is a polycylinder: $N=\{s|| s \mid<1\}$ and $V_{0}=\pi^{-1}(0)$.

v) $W$ is covered by a finite number of coordinate neighborhoods $w_{i}$ such that

$$
V_{0} \cap w_{i}=V_{0} \cap u_{i}
$$

Each $\mathcal{W}_{i}$ is covered by a system of holomorphic coordinates $\left(z_{i}, s\right)$ such that $\pi\left(z_{i}, s\right)=s$ and

$$
w_{i}=\left\{\left(z_{i}, s\right)|| z_{i}|<1,| s \mid<1\right\}
$$

Moreover, on $V_{0} \cap \boldsymbol{w}_{i}=V_{0} \cap u_{i}$, the system of coordinates $\left(z_{i}\right)$ coincides with $\left(\zeta_{i}\right)$, i.e., $\left(z_{i}, 0\right)$ and $\left(\zeta_{i}, 0\right)$ are the same point on $V_{0} \cap \psi_{i}=V_{0} \cap U_{i}$ if and only if $z_{i}^{1}=\zeta_{i}^{1}, \ldots, z_{i}^{n}=\zeta_{i}^{n}$.

vi) $\left(z_{i}, s\right)$ coincides with $\left(z_{k}, s\right)$ if and only if

$$
z_{i}=h_{t k}\left(z_{k}, s\right)
$$

where $h_{u k}\left(z_{k}, s\right)$ is a vector-valued holomorphic function of $\left(z_{k}, s\right)$ defined on $\mathfrak{W}_{k} \cap W_{i}$.

Let

$$
b_{i k}\left(z_{k}\right)=h_{i k}\left(z_{k}, 0\right)
$$

By v) we have

$$
b_{i k}\left(\zeta_{k}\right)=g_{i k}\left(\zeta_{k}, 0\right)
$$

Let

$$
U_{i}=V_{0} \cap w_{i}=V_{0} \cap u_{i}
$$

and let

$$
N_{\varepsilon}=\{s|| s \mid<\varepsilon\},
$$


where $0<\varepsilon<1$. In view of ii) and v) we may write

We may suppose therefore that

$$
\begin{gathered}
u_{i}=U_{i} \times M, \\
\boldsymbol{w}_{i}=U_{i} \times N .
\end{gathered}
$$

$$
\begin{aligned}
& U_{i} \times N_{8} \subset U_{i} \times N=W_{i}, \\
& U_{i}=U_{i} \times M \subset \mathbf{C}^{n} \times M
\end{aligned}
$$

In order to prove our theorem it suffices to construct a holomorphic map $s \rightarrow t=t(s)$ of $N_{\varepsilon}$ into $M$ such that $t(0)=0$ and holomorphic maps

$$
g_{i}:\left(z_{i}, s\right) \rightarrow\left(\zeta_{i}, t\right)=\left(g_{i}\left(z_{i}, s\right), t(s)\right)
$$

of $U_{i} \times N_{\varepsilon}$ into $\mathrm{C}^{n} \times M$ such that $g_{i}\left(z_{i}, 0\right)=z_{i}$ which satisfy the equations

$$
g_{i}\left(h_{i k}\left(z_{k}, 8\right), s\right)=g_{i k}\left(g_{k}\left(z_{k}, s\right), t(s)\right)
$$

whenever $z_{k} \varepsilon U_{k} \cap U_{i}$ and $|s|$ is sufficiently small (or, more precisely, $|s|<\varepsilon\left(z_{k}\right), \varepsilon\left(z_{k}\right)$ being a continuous function of $z_{k}$ defined on $U_{k} \cap U_{i}$ such that $\left.0<\varepsilon\left(z_{k}\right)<\varepsilon\right)$. In fact, let $\left\{U_{i}^{*}\right\}$ be a covering of $V_{0}$ such that the closure of each $U_{i}^{*}$ is a compact subset of $U_{i}$ and such that $\left\{U_{i}^{*} \times N_{\varepsilon}\right\}$ covers $W \mid N_{\varepsilon}=\pi^{-1}\left(N_{\varepsilon}\right)$. Moreover, let $\delta<\varepsilon$ be a sufficiently small positive number and let $g_{i}^{*}$ be the restriction of $g_{i}$ to $U_{i}^{*} \times N_{\delta}$. Since $g_{i}\left(z_{i}, 0\right)=z_{i}$ and $t(0)=0$, we infer that $g_{i}^{*}$ maps $U_{i}^{*} \times N_{\delta}$ into $U_{i} \times M=U_{i}$. Thus $g_{i}^{*}$ is a holomorphic map of $U_{i}^{*} \times N_{\delta}$ into $\vartheta$. Moreover, (1) implies that $g_{i}^{*}$ and $g_{k}^{*}$ coincide on the intersection $U_{i}^{*} \times N_{\delta} \cap U_{k}^{*} \times N_{\delta}$. Consequently the collection $\left\{g_{i}^{*}\right\}$ determines a holomorphic map $g^{*}$ of $\mathcal{W} \mid N_{\delta}=\pi^{-1}\left(N_{\delta}\right)$ into $\vartheta$ which clearly maps each fibre $\pi^{-1}(s)$ of $W \mid N_{\delta}$ biregularly onto the fibre $\varpi^{-1}(t(s))$ of $\vartheta$. This proves our theorem.

Let

$$
t(s)=t_{1}(s)+t_{2}(s)+\cdots+t_{\mu}(s)+\cdots
$$

be the power series expansion of $t(s)$ and let

$$
t^{\mu}(s)=t_{1}(s)+t_{2}(s)+\cdots+t_{\mu}(s)
$$

Moreover, let

$$
g_{i}\left(z_{i}, s\right)=z_{i}+g_{i \mid \mathbf{1}}\left(z_{i}, s\right)+\cdots+g_{i \mid \mu}\left(z_{i}, s\right)+\cdots
$$

be the power series expansion of $g_{i}\left(z_{i}, 8\right)$ and let

$$
g_{i}^{\mu}\left(z_{i}, s\right)=z_{i}+g_{i \mid 1}\left(z_{i}, s\right)+\cdots+g_{i \mid \mu}\left(z_{i}, s\right) .
$$

We remark that $g_{i \mid \mu}\left(z_{i}, s\right)$ is a homogeneous polynomial in $\left(s_{1}, s_{2}, \cdots, s_{l}\right)$ whose coefficients are vector-valued holomorphic functions of $z_{i}$ defined on $\left\{z_{i}|| z_{i} \mid<1\right\}$. For 
any vector-valued holomorphic functions $P(s), Q(s)$ in $\left(s_{1}, s_{2}, \ldots, s_{l}\right)$, we indicate by writing $P(s) \overline{\bar{\mu}} Q(s)$ that the power series expansion of $P(s)-Q(s)$ in $\left(s_{1}, s_{2}, \ldots, s_{l}\right)$ contains no terms of degree $\leqslant \mu$. Clearly $(1)$ is equivalent to the system of congruences

$$
g_{i}^{\mu}\left(h_{i k}\left(z_{k}, s\right), s\right) \equiv g_{i k}\left(g_{k}^{\mu}\left(z_{k}, s\right), t^{\mu}(s)\right), \quad(\mu=0,1,2, \ldots)
$$

Note that the power series expansions of both sides of $(6)_{\mu}$ are well-defined at each point $z_{k} \in U_{k} \cap U_{i}$.

We insert here a remark on the first cohomology group $H^{1}\left(V_{0}, \Theta_{0}\right)$ of $V_{0}$ with coefficients in the sheaf $\Theta_{0}$ of germs of holomorphic vector fields on $V_{0}$. Denote the covering $\left\{U_{i}\right\}$ by $\mathfrak{u}$. Since each $U_{i}$ is a Stein manifold we have the canonical isomorphism (see Cartan [1], Leray [4])

$$
H^{1}\left(V_{0}, \Theta_{0}\right) \cong H^{1}\left(\mathfrak{l l}, \Theta_{0}\right)
$$

Let $\left\{\theta_{i k}\right\}$ be a 1 -cocycle on $\mathfrak{U}=\left\{U_{i}\right\}$ with coefficients in $\Theta_{0}$, i.e., a system of holomorphic vector fields $\theta_{\mathfrak{k} k}$ defined respectively on $U_{i} \cap U_{k}$ such that

$$
\theta_{i k}=\theta_{i j}+\theta_{j k}, \quad \text { on } U_{i} \cap U_{1} \cap U_{k} .
$$

We write $\theta_{i k}$ explicitly in the form

$$
\theta_{i k}\left(z_{i}\right)=\left(\theta_{i k}^{1}\left(z_{i}\right), \ldots, \theta_{i k}^{\alpha}\left(z_{i}\right), \ldots, \theta_{i k}^{n}\left(z_{i}\right)\right)
$$

with reference to the system of coordinates $\left(z_{i}\right)=\left(z_{i}^{1}, \ldots, z_{i}^{\alpha}, \ldots, z_{i}^{n}\right)$. The explicit form of the cocycle condition (8) is:

$$
\theta_{i k}^{\alpha}\left(z_{i}\right)=\theta_{i j}^{\alpha}\left(z_{i}\right)+\sum_{\beta=1}^{n} \frac{\partial b_{i j}^{\alpha}\left(z_{j}\right)}{\partial z_{j}^{\beta}} \cdot \theta_{j k}^{\beta}\left(z_{j}\right),
$$

where $z_{i}=b_{i j}\left(z_{j}\right)$. Using matrix notation we write this in the form

$$
\theta_{i k}\left(z_{i}\right)=\theta_{i j}\left(z_{i}\right)+B_{i j}\left(z_{j}\right) \cdot \theta_{j k}\left(z_{j}\right), \quad\left(z_{i}=b_{i j}\left(z_{j}\right)\right),
$$

where $B_{i f}\left(z_{f}\right)$ denotes the $n \times n$ matrix

$$
B_{i j}\left(z_{j}\right)=\left(\frac{\partial b_{i j}^{\alpha}\left(z_{j}\right)}{\partial z_{j}^{\beta}}\right)_{\substack{\alpha=1,2, \ldots, n \\ \beta=1,2, \ldots, n}}
$$

Letting $\quad \beta_{i k r}\left(z_{i}\right)=\left.\frac{\partial g_{i k}\left(z_{k}, t\right)}{\partial t_{r}}\right|_{t=0}, \quad$ where $z_{i}=b_{i k}\left(z_{k}\right)$,

we obtain a 1-cocycle $\left\{\beta_{i k r}\left(z_{i}\right)\right\}$ on $\mathfrak{U}=\left\{U_{i}\right\}$ with coefficients in $\Theta_{0}$. For any tangent vector 


$$
v=\sum_{r=1}^{m} v_{r} \frac{\partial}{\partial t_{r}}
$$

of $M$ at 0 , the infinitesimal deformation $\varrho_{0}(v) \in H^{1}\left(V_{0}, \Theta_{0}\right)$ is, by definition, the cohomology class of the 1-cocycle

$$
\left\{\sum_{r=1}^{m} v_{r} \beta_{i k r}\left(z_{i}\right)\right\}
$$

By hypothesis, $\underline{o}_{0}:\left(T_{M}\right)_{0} \rightarrow H^{1}\left(V_{0}, \Theta_{0}\right)$ is surjective. In view of the canonical isomorphism (7), we infer therefore that any 1-cocycle $\left\{\theta_{i k}\left(z_{i}\right)\right\}$ is cohomologous to a linear combination of $\left\{\beta_{i k r}\left(z_{i}\right)\right\}, r=1,2, \ldots, m$. In other words, for any 1-cocycle $\left\{\theta_{i k}\left(z_{i}\right)\right\}$, we can find constants $\gamma_{1}, \ldots, \gamma_{r}, \ldots, \gamma_{m}$ and holomorphic vector fields

$$
\theta_{i}\left(z_{i}\right)=\left(\theta_{i}^{1}\left(z_{i}\right), \ldots, \theta_{i}^{\alpha}\left(z_{i}\right), \ldots, \theta_{i}^{n}\left(z_{i}\right)\right)
$$

defined respectively on $U_{i}$ such that

$$
\sum_{r=1}^{m} \gamma_{r} \beta_{i k r}\left(z_{i}\right)+B_{i k}\left(z_{k}\right) \cdot \theta_{k}\left(z_{k}\right)-\theta_{i}\left(z_{i}\right)=\theta_{i k}\left(z_{i}\right)
$$

where $z_{i}=b_{i k}\left(z_{k}\right)$.

We may assume that $\beta_{i k r}\left(z_{i}\right)$ and $B_{i k}\left(z_{k}\right)$ are uniformly bounded:

$$
\left|\beta_{i k r}\left(z_{i}\right)\right|<K_{1}, \quad\left|B_{i k}\left(z_{k}\right)\right|<K_{1},
$$

where $\left|B_{i k}\left(z_{k}\right)\right|$ denotes the usual norm of the matrix $B_{i k}\left(z_{k}\right)$. For any 1-cocycle $\sigma=\left\{\theta_{i k}\left(z_{i}\right)\right\}$, we define the norm $\|\sigma\|$ of $\sigma$ by

$$
\|\sigma\|=\max _{i, k} \sup _{z_{i}}\left|\theta_{i k}\left(z_{i}\right)\right|
$$

L м M A 1. For any 1-cocycle $\sigma=\left\{\theta_{i k}\left(z_{i}\right)\right\}$, we can find $\gamma_{r}$ and $\theta_{l}\left(z_{i}\right)$ satisfying (10) such that

$$
\left|\gamma_{r}\right|<K_{2} \cdot\|\sigma\|, \quad\left|\theta_{i}\left(z_{1}\right)\right|<K_{2} \cdot\|\sigma\|
$$

where $K_{2}$ is a positive constant which is independent of $\sigma$.

Proof. We define

$$
\iota(\sigma)=\inf \max \left\{\left|\gamma_{r}\right|, \sup _{z_{i}}\left|\theta_{i}\left(z_{i}\right)\right|\right\}
$$

where inf is taken with respect to all solutions $\left\{\gamma_{r}, \theta_{i}\left(z_{i}\right)\right\}$ of the equations $(10)$. It suffices to prove the existence of a constant $K_{2}$ such that

$$
\iota(\sigma)<K_{2} \cdot\|\sigma\|
$$


Suppose that such a constant $K_{2}$ does not exist. Then we can find a sequence $\sigma^{(1)}, \sigma^{(2)}, \ldots, \sigma^{(v)}, \ldots$ of 1-cocycles $\sigma^{(v)}=\left\{\theta_{i k}^{(v)}\left(z_{i}\right)\right\}$ such that

$$
\iota\left(\sigma^{(v)}\right)=1, \quad\left\|\sigma^{(v)}\right\|<\frac{1}{v}
$$

$\iota\left(\sigma^{(v)}\right)=1$ implies that there exist $\gamma_{r}^{(v)}, \theta_{i}^{(v)}\left(z_{i}\right)$ satisfying

$$
\begin{gathered}
\sum_{r=1}^{m} \gamma_{r}^{(v)} \beta_{i k r}\left(z_{i}\right)+B_{i k}\left(z_{k}\right) \theta_{k}^{(v)}\left(z_{k}\right)-\theta_{i}^{(v)}\left(z_{i}\right)=\theta_{i k}^{(v)}\left(z_{i}\right) \\
\left|\gamma_{r}^{(v)}\right|<2, \quad\left|\theta_{i}^{(v)}\left(z_{i}\right)\right|<2,
\end{gathered}
$$

where $z_{i}=b_{i k}\left(z_{k}\right)$. Hence, replacing $\sigma^{(1)}, \sigma^{(2)}, \ldots$ by a suitable subsequence if necessary, we may suppose that

$$
\begin{gathered}
\gamma_{r}=\lim _{v \rightarrow \infty} \gamma_{r}^{(v)}, \\
\theta_{i}\left(z_{i}\right)=\lim _{v \rightarrow \infty} \theta_{i}^{(v)}\left(z_{i}\right)
\end{gathered}
$$

exist, where the convergence $\theta_{i}^{(v)}\left(z_{i}\right) \rightarrow \theta_{i}\left(z_{i}\right)$ is uniform on each compact subset of $U_{i}$ and $\theta_{i}\left(z_{i}\right)$ is holomorphic on $U_{i}$. Since

$$
\left|\theta_{i k}^{(v)}\left(z_{i}\right)\right| \leqslant\left\|\sigma^{(\nu)}\right\| \rightarrow 0(\nu \rightarrow \infty)
$$

we obtain from (13) the equality

$$
\sum_{r=1}^{m} \gamma_{r} \beta_{i k r}\left(z_{i}\right)+B_{i k}\left(z_{k}\right) \theta_{k}\left(z_{k}\right)-\theta_{i}\left(z_{i}\right)=0
$$

Let $\left\{U_{i}^{*}\right\}$ be a covering of $V_{0}$ such that the closure of each $U_{i}^{*}$ is a compact subset of $U_{i}$. For each point $z_{i} \in U_{i}$ there exists at least one $U_{k}^{*}$ which contains $z_{k}=b_{k i}\left(z_{i}\right)$. Hence we infer from (13) and (15) that $\theta_{i}^{(p)}\left(z_{i}\right)$ converges to $\theta_{i}\left(z_{i}\right)$ uniformly on the whole of $U_{i}$.

Letting

$$
\gamma_{r}^{\prime}=\gamma_{r}^{(v)}-\gamma_{r}, \quad \theta_{i}^{\prime}\left(z_{i}\right)=\theta_{i}^{(\nu)}\left(z_{i}\right)-\theta_{i}\left(z_{i}\right)
$$

for a sufficiently larger integer $v$, we have therefore

$$
\left|\gamma_{r}^{\prime}\right|<\frac{1}{2}, \quad\left|\theta_{i}^{\prime}\left(z_{i}\right)\right|<\frac{1}{2}
$$

while we infer from (13) and (16) that 


$$
\sum_{r=1}^{m} \gamma_{r}^{\prime} \beta_{i k r}\left(z_{i}\right)+B_{i k}\left(z_{k}\right) \theta_{k}^{\prime}\left(z_{k}\right)-\theta_{i}^{\prime}\left(z_{i}\right)=\theta_{i k}^{(y)}\left(z_{i}\right)
$$

This contradicts with $\iota\left(\sigma^{(\nu)}\right)=1$, q.e.d.

Now we construct $t^{\mu}(s)$ and $g_{i}^{\mu}\left(z_{i}, s\right)$ satisfying $(6)_{\mu}$ by induction on $\mu$. It follows from the identity

$$
h_{i k}\left(z_{k}, 0\right)=b_{i k}(z)=g_{i k}\left(z_{k}, 0\right)
$$

that $t^{0}(s)=0$ and $g_{i}^{0}\left(z_{i}, s\right)=z_{i}$ satisfy $(6)_{0}$. Suppose therefore that $t^{\mu-1}(s)$ and $g_{i}^{\mu-1}\left(z_{i}, s\right)$ satisfying $(6)_{\mu-1}$ are already determined. We define a homogeneous polynomial $\Gamma_{i k \mid \mu}\left(z_{i}, s\right)$ of degree $\mu$ in $\left(s_{1}, s_{2}, \ldots, s_{l}\right)$, whose coefficients are vector-valued holomorphic functions of $z_{i}$ defined on $U_{i} \cap U_{k}$, by the congruence

$$
\Gamma_{i k \mid \mu}\left(z_{i}, s\right) \equiv g_{i}^{\mu-1}\left(h_{i k}\left(z_{k}, s\right), s\right)-g_{i k}\left(g_{k}^{\mu-1}\left(z_{k}, s\right), t^{\mu-1}(s)\right),
$$

where $z_{i}=b_{i k}\left(z_{k}\right)$.

L $\mathbf{M}$ M A 2. We have the identity

$$
\Gamma_{i k \mid \mu}\left(z_{i}, s\right)=\Gamma_{i j \mid \mu}\left(z_{i}, s\right)+B_{i j}\left(z_{j}\right) \cdot \Gamma_{j k \mid \mu}\left(z_{j}, s\right)
$$

where $z_{i}=b_{i j}\left(z_{j}\right)$.

Proof. For simplicity let $\Gamma_{i k \mid \mu}=\Gamma_{i k \mid \mu}\left(z_{i}, s\right), \Gamma_{i j \mid \mu}=\Gamma_{i j \mid \mu}\left(z_{i}, s\right)$ and $\Gamma_{j k \mid \mu}=\Gamma_{j k \mid \mu}\left(z_{j}, s\right)$, where $z_{i}=b_{i j}\left(z_{j}\right)=b_{i k}\left(z_{k}\right), z_{j}=b_{\text {gk }}\left(z_{k}\right)$. Since

$$
g_{i k}\left(z_{k}, t\right)=g_{i j}\left(g_{j k}\left(z_{k}, t\right), t\right) \text {, }
$$

we have

$$
\Gamma_{i k \mid \mu} \equiv g_{\mu}^{\mu-1}\left(h_{i k}\left(z_{k}, s\right), s\right)-g_{i j}\left(g_{j k}\left(g_{k}^{\mu-1}\left(z_{k}, s\right), t^{\mu-1}(s)\right), t^{\mu-1}(s)\right)
$$

Using

$$
g_{j k}\left(g_{k}^{\mu-1}\left(z_{k}, s\right), t^{\mu-1}(s)\right) \equiv{ }_{\mu} g_{j}^{\mu-1}\left(h_{j k}\left(z_{k}, s\right), s\right)-\Gamma_{j k \mid \mu},
$$

we get

$$
g_{i j}\left(g_{j k}\left(g_{k}^{\mu-1}\left(z_{k}, s\right), t^{\mu-1}(s)\right), t^{\mu-1}(s)\right) \underset{\mu}{\equiv} g_{i j}\left(g^{\mu-1}\left(h_{j k}\left(z_{k}, s\right), s\right), t^{\mu-1}(s)\right)-B_{i j}\left(z_{j}\right) \cdot \Gamma_{j k \mid \mu}
$$

since

$$
g_{j}^{\mu-1}\left(h_{j k}\left(z_{k}, 0\right), 0\right)=b_{j k}\left(z_{k}\right)=z_{j}
$$

Hence we obtain

$$
\Gamma_{i k \mid \mu} \equiv g_{\mu}^{\mu-1}\left(h_{i k}\left(z_{k}, s\right), s\right)-g_{i j}\left(g_{j}^{\mu-1}\left(h_{j k}\left(z_{k}, s\right), s\right), t^{\mu-1}(s)\right)+B_{i j}\left(z_{j}\right) \cdot \Gamma_{j k \mid \mu}
$$

Now, using $h_{i k}\left(z_{k}, s\right)=h_{i j}\left(h_{j k}\left(z_{k}, s\right), s\right)$, we get 


$$
\begin{aligned}
g_{i}^{\mu-1}\left(h_{i k}\left(z_{k}, s\right), s\right)-g_{i j}\left(g_{j}^{\mu-1}\left(h_{j k}\left(z_{k}, s\right), s\right), t^{\mu-1}(s)\right)=g_{i}^{\mu-1}\left(h_{i j}\left(h_{j k}\left(z_{k}, s\right), s\right), s\right) \\
\quad-g_{i j}\left(g_{j}^{\mu-1}\left(h_{j k}\left(z_{k}, s\right), s\right), t^{\mu-1}(s)\right) \underset{\mu}{\equiv} \Gamma_{i j \mid \mu}\left(b_{i j}\left(h_{j k}\left(z_{k}, s\right)\right), s\right) \equiv \Gamma_{i j ; \mu}\left(b_{i k}\left(z_{k}\right), s\right)
\end{aligned}
$$

Consequently we obtain

$$
\Gamma_{i k \mid \mu} \equiv \Gamma_{i j \mid \mu}+B_{i j}\left(z_{j}\right) \cdot \Gamma_{j k \mid \mu}, \quad \text { q.e.d. }
$$

Our purpose is to determine

$$
t^{\mu}(s)=t^{\mu-1}(s)+t_{\mu}(s), \quad g_{i}^{\mu}\left(z_{i}, s\right)=g_{i}^{\mu-1}\left(z_{i}, s\right)+g_{i \mid \mu}(z, s)
$$

which satisfy $(6)_{\mu}$. Letting

we have

$$
t_{\mu}(s)=\left(t_{1 \mid \mu}(s), \ldots, t_{r \mid \mu}(s), \ldots, t_{m \mid \mu}(s)\right),
$$

$$
\begin{aligned}
g_{i k}\left(g_{k}^{\mu-1}\left(z_{k}, s\right)+g_{k \mid / k}\left(z_{k}, s\right), t^{\mu-1}(s)+t_{\mu}(s)\right) \\
\quad \equiv g_{j k}\left(g_{k}^{\mu-1}\left(z_{k}, s\right), t^{\mu-1}(s)\right)+B_{i k}\left(z_{k}\right) \cdot g_{k \mid \mu}\left(z_{k}, s\right)+\sum_{r=1}^{m} t_{r \mid \mu}(s) \beta_{i k \mid r}\left(z_{j}\right),
\end{aligned}
$$

where $z_{i}=b_{i k}\left(z_{k}\right)$, while

$$
g_{i \mid \mu}\left(h_{i j}\left(z_{k}, s\right), s\right) \equiv g_{i \mid \mu}\left(z_{i}, s\right) .
$$

Therefore, $(6)_{\mu}$ is equivalent to the equalities

$$
\sum_{r=1}^{m} t_{\tau \mid \mu}(s) \beta_{i k \mid \tau}\left(z_{i}\right)+B_{i k}\left(z_{k}\right) \cdot g_{k \mid \mu}\left(z_{k}, s\right)-g_{i \mid \mu}\left(z_{i}, s\right)=\Gamma_{i k \mid \mu}\left(z_{i}, s\right)
$$

Now the formula (17) shows that $\left\{\Gamma_{i k \mid \mu}\left(z_{i}, s\right)\right\}$ is a homogeneous polynomial in $s$ of degree $\mu$ whose coefficients form a 1-cocycle on $\mathfrak{U}=\left\{U_{i}\right\}$ with coefficients in $\Theta_{0}$. Consequently, by the above remark (see (10)), we can find homogeneous polynomials $t_{r \mid \mu}(s)$ with constant coefficients and homogeneous polynomials $g_{i \mid \mu}\left(z_{i}, s\right)$ whose coefficients are vector-valued holomorphic functions on $U_{i}$ which satisfy (18). This completes our inductive construction of $t^{\mu}(s)$ and $g_{i}^{\mu}\left(z_{i}, s\right)$.

Now we prove that, if we choose proper solutions $t_{r \mid \mu}(s), g_{\mid \mu}\left(z_{t}, s\right)$ of the equation (18) in each step of the above construction, the power series

$$
t(s)=t_{1}(s)+t_{2}(s)+\cdots+t_{\mu}(s)+\cdots, \quad g_{i}\left(z_{i}, s\right)=z_{i}+g_{i \mid 1}\left(z_{i}, s\right)+\cdots+g_{i \mid \mu}\left(z_{i}, s\right)+\cdots
$$

converge absolutely and uniformly for $|s|<\varepsilon$ provided that $\varepsilon>0$ is sufficiently small. 
Consider a power series

$$
f(s)=\sum f_{h_{1} h_{3} \ldots h_{l}} s_{1}^{h_{1}} s_{2}^{h_{*}} \ldots s_{l}^{h_{l}}
$$

whose coefficients $f_{h_{1}} h_{1} \ldots h_{l}$ are vectors and a power series

$$
a(s)=\sum a_{h_{1} h_{2} \ldots h_{l}} s_{1}^{h_{1}} s_{2}^{h_{2}} \ldots s_{l}^{h_{l}}
$$

with non-negative coefficients $a_{h_{2} h_{2} \ldots h_{l}}$. We indicate by writing $f(s)<a(s)$ that

$$
\left|f_{h_{1} h_{1} \ldots h_{l}}\right|<a_{h_{1} h_{2} \ldots h_{l}} .
$$

Let

$$
A(s)=\frac{b}{64 c} \sum_{\mu=1}^{\infty} \frac{1}{\mu^{2}} c^{\mu}\left(s_{1}+s_{2}+\cdots+s_{l}\right)^{\mu}
$$

We remark that

$$
A(s)^{\nu}<\left(\frac{b}{c}\right)^{\nu-1} A(8), \quad \nu=2,3,4, \ldots
$$

Let

$$
z_{k}+y=\left(z_{k}^{1}+y_{1}, \ldots, z_{k}^{\alpha}+y_{\alpha}, \ldots, z_{k}^{n}+y_{n}\right)
$$

We may assume that the power series expansion of $g_{i k}\left(z_{k}+y, t\right)$ in $n+m$ variables $y_{1}, \ldots, y_{n}, t_{1}, \ldots, t_{m}$ satisfies

$$
g_{t k}\left(z_{k}+y, t\right)-b_{i k}\left(z_{k}\right)<A_{0}(y, t), \quad z_{k} \in U_{k} \cap U_{t}
$$

where

$$
A_{0}(y, t)=\frac{b_{0}}{c_{0}} \sum_{\mu=1}^{\infty} c_{0}^{\prime \prime}\left(y_{1}+\cdots+y_{n}+t_{1}+\cdots+t_{m}\right)^{\mu}
$$

Moreover, we may assume that

$$
h_{i k}\left(z_{k}, s\right)-b_{i k}\left(z_{k}\right)<A_{0}(s), \quad z_{k} \in U_{k} \cap U_{i}
$$

where $A_{0}(s)$ is the function $A(s)$ in which the constants $b, c$ are replaced by $b_{0}, c_{0}$. For our purpose it suffices to derive the estimates

$$
t^{\mu}(s)<A(s), \quad g_{i}^{\mu}\left(z_{i}, s\right)-z_{i}<A(s)
$$

by induction on $\mu$ provided that the constants $b, c$ are chosen properly. For $\mu=1$ the estimates $(22)_{1}$ are obvious if $b$ is sufficiently large. Assume therefore that estimates $(22)_{\mu-1}$ are established for some $\mu$. We have

$$
\Gamma_{i k \mid \mu}\left(z_{i}, s\right) \underset{\bar{\mu}}{\equiv} g_{i}^{\mu-1}\left(h_{i k}\left(z_{k}, s\right), s\right)-g_{i k}\left(g_{k}^{\mu-1}\left(z_{k}, s\right), t^{\mu-1}(s)\right)
$$


where $z_{i}=b_{i k}\left(z_{k}\right)$. Letting $\quad U_{i}^{\delta}=\left\{z_{i}|| z_{i} \mid<1-\delta\right\}$

we first estimate $\Gamma_{i k \mid \mu}\left(z_{i}, s\right)$ for $z_{i} \in U_{i}^{\delta} \cap U_{k}$, where $\delta$ is a sufficiently small positive number such that $\left\{U_{i}^{\delta}\right\}$ forms a covering of $V_{0}$. Set

$$
G_{i}\left(z_{i}, s\right)=g_{i}^{\mu-1}\left(z_{i}, s\right)-z_{i}
$$

for simplicity and expand $G_{i}\left(z_{i}+y, s\right)$ into power series in $y_{1}, \ldots, y_{n}, s_{1}, \ldots, s_{l}$. Since by our hypothesis,

$$
G_{i}\left(z_{i}, s\right)<A(s), \text { for }\left|z_{i}\right|<1
$$

we get

$$
G_{i}\left(z_{i}+y, s\right)-G_{i}\left(z_{i}, s\right)<A(s) \sum \frac{y_{1}^{y_{1}} y_{2}^{y_{1}} \ldots y_{n}^{y_{n}}}{\delta_{r}^{\nu_{1}+v_{1}+\cdots+y_{n}}}, \quad \text { for }\left|z_{i}\right|<1-\delta
$$

where $\sum$ is extended over all non-negative integers $v_{1}, v_{2}, \ldots, v_{n}$ with $v_{1}+v_{2}+\cdots+v_{n} \geqslant 1$. Letting $y=h_{i k}\left(z_{k}, 8\right)-b_{i k}\left(z_{k}\right), z_{i}=b_{i k}\left(z_{k}\right)$ and using (21), we obtain from this

$$
G_{i}\left(h_{i k}\left(z_{k}, s\right), s\right)-G_{i}\left(z_{i}, s\right)<A(s)\left\{\left(\sum_{v=0}^{\infty} \delta^{-r} A_{0}(s)^{p}\right)^{n}-1\right\}, \quad \text { for } z_{i} \in U_{i}^{\delta} \cap U_{k} .
$$

Since $A_{0}(s)^{p}<\left(b_{0} / c_{0}\right)^{p-1} A_{0}(s)$ for $\nu \geqslant 2$, we have

$$
\frac{A_{0}(s)^{\nu}}{\delta^{\nu}} \ll\left(\frac{b_{0}}{c_{0} \delta}\right)^{\nu-1} \cdot \frac{A_{0}(s)}{\delta}, \text { for } \nu \geqslant 2
$$

We may assume that

$$
\frac{b_{0}}{c_{0} \delta}<\frac{1}{2}
$$

since (20) and (21) remain valid if we replace $c_{0}$ by a larger constant. Hence we have

$$
\frac{A_{0}(s)^{y}}{\delta^{\nu}}<\frac{A_{0}(s)}{2^{v-1} \delta}, \text { for } \nu \geqslant 2 .
$$

Using this we obtain

or

$$
G_{i}\left(h_{i k}\left(z_{k}, s\right), s\right)-G_{i}\left(z_{i}, s\right) \ll A(s)\left\{\left(1+\frac{2 A_{0}(s)}{\delta}\right)^{n}-1\right\}<\frac{K_{0}}{\delta} A(s) A_{0}(s),
$$

$$
g_{i}^{\mu-1}\left(h_{i k}\left(z_{k}, s\right), s\right)-h_{i k}\left(z_{k}, s\right)-g_{i}^{\mu-1}\left(z_{i}, s\right)+z_{i}<\frac{K_{0}}{\delta} A(s) A_{0}(s), \text { for } z_{i} \in U_{i}^{d} \cap U_{k},
$$

where $z_{i}=b_{i k}\left(z_{k}\right)$ and where $K_{0}$ is a constant depending only on $n$. Assuming that

$$
b=b_{0}, \quad c>c_{0},
$$


we have

$$
A_{0}(s) \ll \frac{b_{0}}{b} \cdot A_{0}(s)
$$

and therefore

$$
A(s) A_{0}(s) \ll \frac{b_{0}}{b} A(s)^{2} \ll \frac{b_{0}}{c} A(s)
$$

Consequently we infer from (24) and (21) that

$$
g_{i}^{\mu-1}\left(h_{i k}\left(z_{k}, s\right), s\right)-g_{i}^{\mu-1}\left(z_{i}, s\right)<\left(\frac{K_{0} b_{0}}{\delta c}+\frac{b_{0}}{b}\right) A(s), \quad \text { for } z_{i} \in U_{\mathfrak{i}}^{\delta} \cap U_{k} \text {. }
$$

For any power series

$$
f(s)=f_{0}+f_{1}(s)+\cdots+f_{\mu}(s)+\cdots
$$

we denote by $[f(s)]_{\mu}$ the term $f_{\mu}(s)$ of degree $\mu$. Then we get from (26)

$$
\left[g_{t}^{\mu-1}\left(h_{i k}\left(z_{k}, s\right), s\right)\right]_{\mu}<\left(\frac{K_{0} b_{0}}{\delta c}+\frac{b_{0}}{b}\right) A(s) \quad \text { for } z_{k} \in U_{k} \cap U_{i}^{\delta}
$$

Next we estimate $g_{i k}\left(g_{k}^{\mu-1}\left(z_{k}, s\right), t^{\mu-1}(s)\right)$. We expand $g_{i k}\left(z_{k}+y, t\right)$ into power series in $y_{1}, \ldots, y_{n}, t_{1}, \ldots, t_{m}$ and let

$$
L_{i k}\left(z_{k}, y, t\right)=\left[g_{i k}\left(z_{k}+y, t\right)\right]_{1}
$$

be the linear term of the power series. Then we have, by (20),

$$
g_{i k}\left(z_{k}+y, t\right)-b_{t k}\left(z_{k}\right)-L_{t k}\left(z_{k}, y, t\right)<\frac{b_{0}}{c_{0}} \sum_{\mu=2}^{\infty} c_{0}^{\mu}\left(y_{1}+\cdots+y_{n}+t_{1}+\cdots+t_{m}\right)^{\mu}
$$

Letting $y=g_{k}^{\mu-1}\left(z_{k}, s\right)-z_{k}, t=t^{\mu-1}(s)$ and using our inductive hypothesis $(22)_{\mu-1}$, we obtain from this the estimate

$$
\left[g_{i k}\left(g_{k}^{\mu-1}\left(z_{k}, s\right), t^{\mu-1}(s)\right)\right]_{\mu} \ll \frac{b_{0}}{c_{0}} \sum_{\mu-2}^{\infty} c_{0}^{\mu}(m+n)^{\mu} A(s)^{\mu} .
$$

Assume that

$$
\frac{(m+n) b c_{0}}{c}<\frac{1}{2}
$$

Then we have

$$
\sum_{\mu=2}^{\infty} c_{0}^{\mu}(m+n)^{\mu} A(s)^{\mu} \ll \sum_{\mu=2}^{\infty} c_{0}^{\mu}(m+n)^{p}\left(\frac{b}{c}\right)^{\mu-1} A(s)<\frac{2(m+n)^{2} b c_{0}^{2}}{c} \cdot A(s)
$$

and therefore $\quad\left[g_{i k}\left(g_{k}^{\mu-1}\left(z_{k}, s\right), t^{\mu-1}(s)\right)\right]_{\mu}<\frac{2(m+n)^{2} b b_{0} c_{0}}{c} \cdot A(s)$. 
Combining this with (27) we obtain

where

$$
\Gamma_{i k\} \mu}\left(z_{i}, s\right)<c^{*} \cdot A(s), \quad \text { for } z_{i} \in U_{i}^{\delta} \cap U_{k},
$$

$$
c^{*}=\frac{K_{0} b_{0}}{\delta c}+\frac{b_{0}}{b}+\frac{2(m+n)^{2} b b_{0} c_{0}}{c} .
$$

Now we recall that the $\Gamma_{i k \mid \mu}\left(z_{i}, s\right)$ satisfy the cocycle condition (17). In particular we have

$$
\Gamma_{i k \mid \mu}\left(z_{i}, s\right)=B_{i k}\left(z_{k}\right) \cdot \Gamma_{k i \mid \mu}\left(z_{k}, s\right)
$$

Combining this with (11) and (29) we get

$$
\Gamma_{i k i \mu}\left(z_{i}, s\right)<c^{*} K_{1} A(s), \text { for } z_{i} \in U_{i} \cap U_{k}^{\delta} .
$$

For an arbitrary point $z_{i} \in U_{i} \cap U_{k}$ there exists one $U_{j}^{\delta}$ which contains $z_{i}$. Therefore we infer from (17), (29) and (30) that

$$
\Gamma_{i k_{i} \mu}\left(z_{i}, s\right) \ll 2 c^{*} K_{1} A(s),
$$

and consequently, by Lemma 1 , we can choose solutions $t_{r \mid \mu}(s)$ and $g_{t \mid \mu}\left(z_{i}, s\right)$ of the equations (18) such that

$$
t_{\mu}(s)<2 c^{*} K_{1} K_{2} A(s), \quad g_{i \mid \mu}\left(z_{i}, s\right)<2 c^{*} K_{1} K_{2} A(s) .
$$

On the other hand, it is clear that, by a proper choice of the constants $b$ and $c$ satisfying our requirements (25), (28), we obtain

$$
2 c^{*} K_{1} K_{2}<1
$$

Consequently we obtain

$$
t_{\mu}(s) \ll A(s), \quad g_{i] \mu}\left(z_{i}, s\right) \ll A(s) .
$$

This proves $(22)_{\mu}$, q.e.d.

\section{References}

[1]. H. Cartan, "Variétés analytiques complexes et cohomologie". Colloque sur les fonctions des plusieurs variables tenu à Bruxelles, 1953, 41-55. Georges Thone, Liège; Masson et Cie, Paris, 1953.

[2]. K. Kodatra, "A theorem of completeness for differentiable families of compact complex manifolds" (to appear).

[3]. K. Kodaira \& D. C. Spencer, “On deformations of complex analytic structures, I-II". Ann. of Math., 67 (1958), 328-466.

[4]. J. LERAY, 'L'anneau spectral et l'anneau filtré d'homologie d'un espace locakement com. pact et d'une application continue". J. Math. Pures Appl., 29 (1950), 1-139. 